\title{
Characterization of microstructure and fatigue life of CP titanium grade 2 specimens subject to various bending processes
}

\author{
H. Fidder ${ }^{1}$, A. Botes ${ }^{1}$, S. Woudberg ${ }^{2}$ \& P. J. McGrath ${ }^{1}$ \\ ${ }^{1}$ Department of Mechanical Engineering, \\ Nelson Mandela Metropolitan University, South Africa \\ ${ }^{2}$ Applied Mathematics Division, Department of Mathematical Sciences, \\ Stellenbosch University, South Africa
}

\begin{abstract}
This study focussed on an investigation into the fatigue behaviour of commercially pure (CP) titanium (Ti) grade 2 specimens produced by various bending (deformation) processes, i.e. mechanical bending, laser bending and laser-mechanical bending. Although published literature on the fatigue behaviour of components produced by laser forming is relatively limited, it was found that laser bending could improve the mechanical behaviour of such components. At high fatigue load settings the microstructure was the dominant contributor to crack initiation and crack growth. After the specimens were bent, residual stress analysis as well as a characterization of the microstructure was performed on the three bent samples as well as the parent plate. Mathematical equations were formulated and presented for the laser forming process for predicting the fatigue life of CP Ti grade 2. This study revealed that the laser forming process can be successfully used as a manufacturing method in the forming of CP Ti grade 2.

Keywords: laser forming, fatigue testing, residual stress, commercially pure titanium, microstructure.
\end{abstract}

\section{Introduction}

When a material is subjected to cyclic loading, progressive and localised damage occurs in the material with time. These loading cycles, measured in stress values, are lower than the (ultimate) tensile strength and often below the tensile yield 
strength of the material. Cyclic loading can be described as repeated loading and unloading of a component and could lead to microscopic cracks developing on the surface. When these cracks develop to a critical size through the load bearing cross-section, sudden failure of a structure can occur. A significant influence on fatigue life is the shape of the component which can amplify concentrated stress levels (ASM International [1, 2]).

This study was aimed at describing the fatigue behaviour of CP Ti grade 2 specimens (3.2mm thick) produced by three different forming methods. The three processes investigated were: a) mechanical bending; b) laser bending and; c) laser-mechanical bending. Few literary sources exist describing the fatigue behaviour of components produced by laser forming and the possibility exists that this forming method could lead to an improvement in the fatigue behaviour of components.

From literature it has been identified that the grain size of unalloyed titanium has a significant effect on the number of cycles to failure [2]. The crack initiation and crack growth mechanism as well as the surface residual stress magnitudes are additional factors that significantly influence the fatigue life of CP Ti grade 2. This experimental study was performed to measure the residual stress magnitudes of the various formed samples together with microstructure evaluation in order to determine their influence on the resultant fatigue life of samples produced by the different forming processes.

\section{Experimental setup}

\subsection{Forming processes}

All specimens were deformed to a radius of curvature of approximately $120 \mathrm{~mm}$. Mechanical forming was performed on a RajalGamete mechanical bending press capable of a maximum force of 25 tons. Laser forming was done using a continuous wave $5 \mathrm{~kW} \mathrm{CO}$ Trump laser system with a doughnut shaped laser beam. Because of the laser system's long wavelength $(10.6 \mu \mathrm{m})$ the sample surfaces were coated with a matt black spray to ensure maximum laser beam absorptive. Although burn-off was evident after the first scan the irradiated surfaces remained sufficiently dark to prevent reflection of the laser beam. The specific laser settings used to produce the laser formed samples was a $1.4 \mathrm{~kW}$ laser power; $12 \mathrm{~mm}$ laser beam diameter; $1.2 \mathrm{~m} / \mathrm{min}$ scanning velocity. The number of scans per line was six with a $6 \mathrm{~mm}$ interval spacing between consecutive lines scans i.e. a 50\% overlap between lines.

Laser-mechanical formed samples were produced by first laser forming to an average radius of curvature of $240 \mathrm{~mm}$ after which it was mechanically formed to an average radius of $120 \mathrm{~mm}$. Plate samples of size $200 \times 50 \times 3 \mathrm{~mm}$ were prepared for laser and mechanical forming. 


\subsection{Fatigue}

The dimensions of the fatigue specimens were based on details given in ASTM E466-96 [3]. After cleaning the samples with a deburring tool and acetone, the specimens were coated with a non-conductive primer where after conductive paint was used to replicate a circuit on the specimen to ensure that the machine switches off once crack initiation commences.

\subsection{Residual stress}

Residual stress measurements were performed using the hole drilling method. An EA-06-062RE-120 strain gauge was used and the principal stresses were calculated using ASTM E837-01 [4]. The drilling depth was set to $2 \mathrm{~mm}$. Residual stress measurements were recorded for the inner and outer radius of the specimens.

\subsection{Microstructure}

Sections for microstructure evaluation were removed from the cross-section of the formed sample at a position that corresponds to the center of the fatigue specimens. The samples were prepared by following standard metallographic procedures and etched in Kroll's reagent to reveal the microstructure.

\section{Results and discussion}

\subsection{Residual stress}

The stress relaxation curves as a function of drilled hole depth for measurements taken at the inner radius of curvature, is shown in fig. 1.

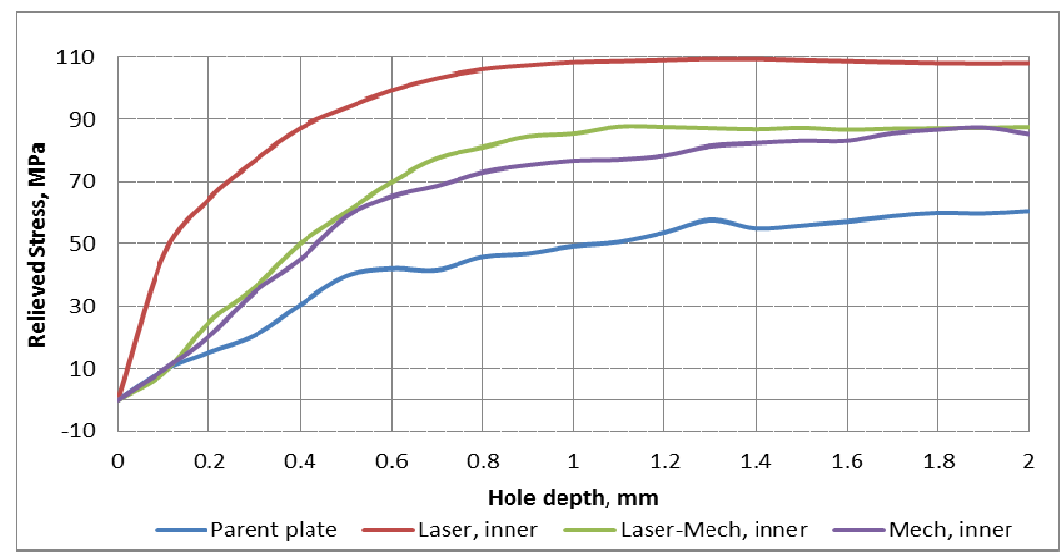

Figure 1: $\quad$ Relieved residual stress of parent plate and formed samples (inner radius of curvature). 
The parent plate sample's curve is given as a reference to indicate the material's residual stress state in the as-received condition. From fig. 1 it can be observed that the laser formed specimen has the highest relieved stress values at all depths compared to the other forming processes. Because the laser forming process takes place with the aid of thermal stresses, a higher residual stress value can be expected. However, when considering the outer radius of curvature, the laser formed specimens yielded similar relieved stress values than the parent plate. The average value was significantly less than what was obtained on the inner radius of curvature, but still higher than the average values obtained for the mechanical and laser-mechanical forming processes. The relieved residual stress values obtained at the inner radius of curvature is thus regarded as the "worst case scenario" and therefore Table 1 only gives the residual stress data measured at the inner radius of curvature for the three forming processes. It is evident from table 1 that the laser forming specimens yielded the highest maximum residual stress value when compared to the other two forming processes. In general all the forming processes lead to an increase in maximum residual stress value as compared to the as-received parent plate.

Table 1: $\quad$ Maximum relieved residual stress values (measured at the inner radius of curvature).

\begin{tabular}{|c|c|}
\hline Specimen & Maximum Relieved Stress (MPa) \\
\hline Parent Plate & 69.15 \\
\hline Laser Formed & 108.25 \\
\hline Mechanical Formed & 87.44 \\
\hline Laser-Mechanical Formed & 87.86 \\
\hline
\end{tabular}

\subsection{Fatigue}

According to literature (ASM International [5]), the S-N (i.e. stress vs. number of cycles) curve for $\mathrm{CP} \mathrm{Ti}$ grade 2 exhibits a distinctive levelling at approximately $10^{5}$ cycles. Therefore, a steeper decrease in fatigue life is observed between $10^{4}$ and $10^{5}$ cycles as opposed to the range of $10^{5}$ to $10^{7}$ cycles. Radonovich [6] also encountered this phenomenon, whereby the transition from high cycle fatigue to low cycle fatigue differs in gradient. Table 2 gives the number of cycles to failure at high, medium and low fatigue load settings for the parent plate and the three forming processes.

Table 2: $\quad$ Results of fatigue testing.

\begin{tabular}{|c|c|c|c|c|}
\hline Load (MPa) & Parent Plate & $\begin{array}{c}\text { Laser } \\
\text { Formed }\end{array}$ & $\begin{array}{c}\text { Mech. } \\
\text { Formed }\end{array}$ & $\begin{array}{c}\text { Laser-Mech. } \\
\text { Formed }\end{array}$ \\
\hline 350.0 (High) & 45420 & 13896 & 11657 & 13382 \\
\hline 241.5 (Med.) & 66457 & 36526 & 48772 & 42600 \\
\hline $\mathbf{2 3 1 . 0}$ (Low) & 88115 & 38506 & 63434 & 55998 \\
\hline
\end{tabular}


Considering all the forming processes, the laser formed specimens performed slightly better (3.8\% better than Laser-Mech. Formed specimens) at the highest load setting but it performed the worst at the medium and low load settings.

\subsection{Microstructure}

The original microstructure of CP Ti grade 2 as-received plate is shown in fig. 2A. The microstructure consists of equiaxedalpha grains with an average grain diameter of $83.4 \mu \mathrm{m}$. Fig. $2 \mathrm{~B}$ shows the microstructure of the laser formed specimens which exhibited a Widmanstätten-like structure throughout the thickness of the specimens. This microstructure for pure titanium is associated with fast cooling rates typically between $800^{\circ} \mathrm{C}$ to $25^{\circ} \mathrm{C}$ via quenching [2]. The microstructure at higher magnification near the laser irradiated surface is shown in fig. 3C. It is evident that small precipitated particles are present in the microstructure.

The cooling rate associated with the laser forming of these specimens was recorded using an infrared thermal camera and the highest cooling rate was obtained during the first line scan corresponding to a cooling rate of $2320.5^{\circ} \mathrm{C} / \mathrm{s}$ whereas the cooling rate for subsequent scan lines were less severe and corresponded to a value of approximately $110^{\circ} \mathrm{C} / \mathrm{s}$ between subsequent scan lines [7].
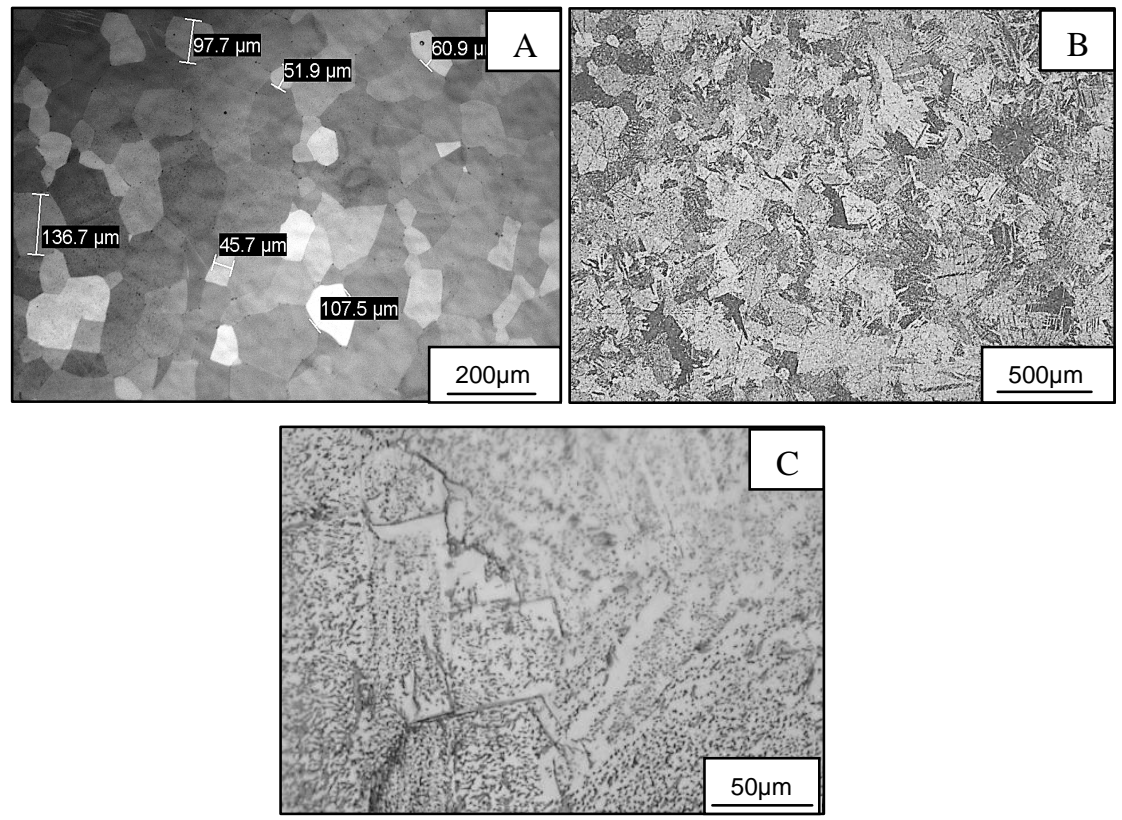

Figure 2: A, original microstructure; $\mathrm{B}$, microstructure of laser formed specimens; and $\mathrm{C}$, near surface microstructure of laser formed $\mathrm{CP}$ Ti grade 2 specimens. 
Fig. 3A shows the microstructure of the mechanical formed specimens which shows clear evidence of twinning at both the inner and outer surface of the radius of curvature. Twinning was observed up to a depth of approximately $257.5 \mu \mathrm{m}$ from the outer radius surface and to a depth of $502.7 \mu \mathrm{m}$ from the inner radius surface. Fig. 3B shows the near surface microstructure of the combined lasermechanical process (laser irradiated side i.e. inner radius of curvature). The Widmanstätten-like structure was observed up to a depth of approximately $312.5 \mu \mathrm{m}$ at the irradiated side while twinning was found to a depth of approximately $261.6 \mu \mathrm{m}$ below the outer radius of curvature surface.

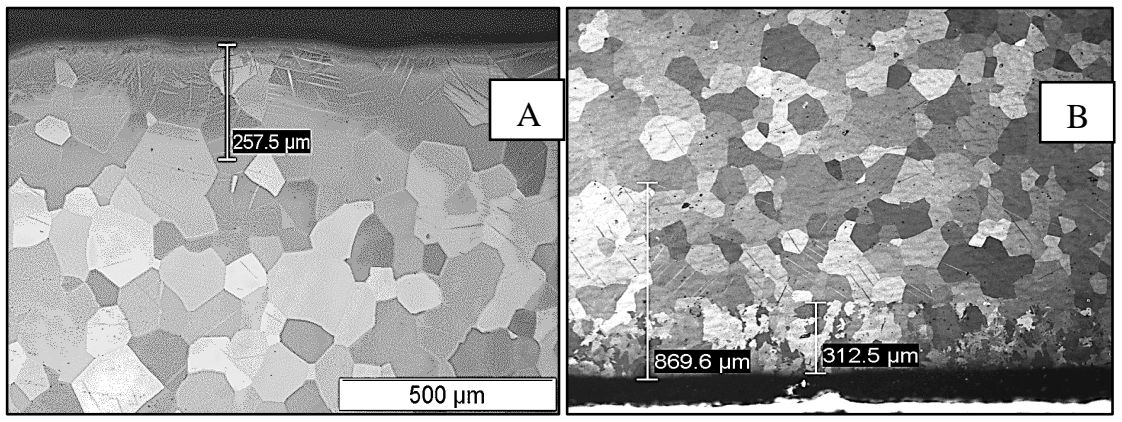

Figure 3: A, microstructure of mechanically formed CP Ti grade 2, and B, microstructure of laser-mechanical formed CP Ti grade 2.

\subsection{Fatigue models}

In order to obtain a mathematical model that can predict the dependence of the fatigue life on the number of cycles to failure, as obtained experimentally for CP Ti grade 2, the power addition technique of Churchill and Usagi [8] is used. The Gerber model (Bannantine et al. [9]) is used as an upper limit and the Goodman model (Bannantine et al. [9]) as a lower limit. Regression curves for the Gerber, Goodman and the actual data were obtained and the residual stress values were incorporated into these models. The power addition technique was first applied to the Gerber model and the curve representing the actual data. The resulting equation was then used together with the Goodman model in the power addition technique, yielding:

$$
y=\left[\left(\left[\left(y_{\text {Gerber }}\right)^{s}+\left(y_{\text {actual }}\right)^{s}\right]^{1 / s}\right)^{-t}+\left(y_{\text {Goodman }}\right)^{-t}\right]^{-1 / t}
$$

The shifting exponents $\boldsymbol{s}$ and $\boldsymbol{t}$ determines the rate at whichthe cross-over takes place between the two asymptotic limits. The higher the value of the shifting parameters, the closer the resulting curve approaches the point of intersection of the two limits. The value of $\boldsymbol{s}$ and $\boldsymbol{t}$ that provided sufficient correspondence with the experimental data was 50 . The resulting prototype predictive equation for the fatigue life of CP Ti grade 2, subject to laser forming, is given by: 


$$
\begin{aligned}
& y=\left[\left(\left[(-0.00075 x+352)^{50}+\left(440 e^{-1.6 \times 10^{-5} x}\right)^{50}\right]^{1 / 50}\right)^{-50}+\right. \\
& \left.(-0.0009 x 259.74)^{-50}\right]^{-1 / 50}
\end{aligned}
$$

Eqn (2) as well as the Gerber and Goodman models are represented graphically in fig. 3. In eqn (2), $\boldsymbol{y}$ denotes the fatigue life and $\boldsymbol{x}$ the number of cycles to failure. By following the same procedure as above, similar eqns can be obtained for the parent plate, mechanical and laser-mechanical forming processes. Since a primary focus of this study is laser forming, the predictive fatigue life equations and corresponding figures for the parent plate and the mechanical and laser-mechanical bending processes are omitted.

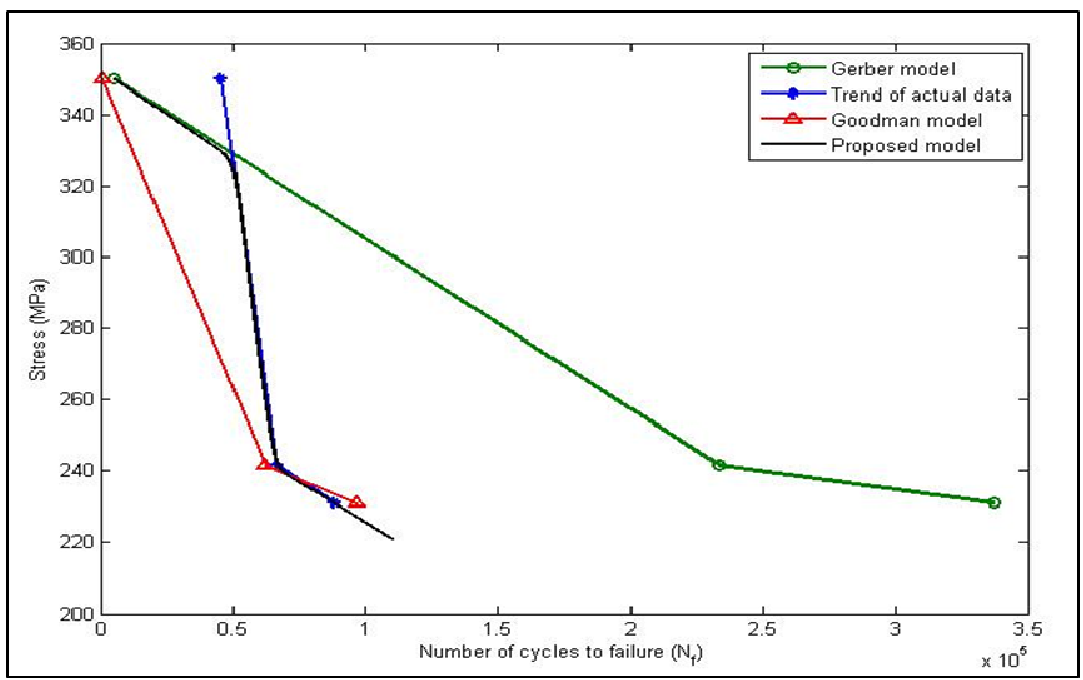

Figure 4: $\quad$ Prototype prediction curve for laser forming.

\section{Conclusions}

It was found that the primary factor influencing the fatigue life of CP Ti grade 2 specimens was the microstructure with the secondary factor being the residual stress, especially at medium to low fatigue load settings. Laser forming resulted in microstructural changes from equiaxed grains to a Widmanstätten structure due to the fast cooling rates experienced during the forming process.

Laser formed samples also revealed the highest values in relieved residual stress whereas the other two processes, i.e. mechanical and laser-mechanical forming, exhibited an increase midway between the parent plate and laser formed samples. 
Fatigue testing revealed that samples produced by laser forming performed the best at the high fatigue load setting even though it performed only marginally (3.8\%) better than the Laser-Mechanical formed specimens. This slight improvement in life cycles at high load settings can be due to the fact that no twinning was observed after forming; therefore crack initiation was slower to develop.

When considering the low fatigue load setting, it can be observed that crack propagation develops relatively quickly within the Widmanstätten structure (at the surface). This phenomenon was also observed by other researchers (Matthew and Donachie - ASM International [2]) where the equiaxed microstructure achieved more cycles before failure than that of the lamellar microstructure. However, it should be noted that the samples were laser formed without any deliberate protective gas shielding. The possibility therefore exists that oxygen and hydrogen diffused into the metal surfaces due to their high solubility in CP Ti. At elevated temperatures hydrides form as hydrogen diffuses into the material during exposure with either gaseous or cathodic hydrogen. Hydrides cause a loss in ductility and reduce the intensity threshold for crack propagation [2].

The proposed mathematical model for predicting the fatigue life of $\mathrm{CP} \mathrm{Ti}$ grade 2 subject to laser forming incorporates existing fatigue models as well as the measured experimental data which can be used for further analysis in understanding the behaviour of this material.

\section{Acknowledgements}

This work would not have been achieved without the assistance of the personnel at the National Laser Centre facility at the CSIR and Mr G Erasmus from the Department of Mechanical Engineering at the Nelson Mandela Metropolitan University for laboratory and technical assistance.

This material is based upon work supported financially by the National Research Foundation. Any opinion, findings and conclusions or recommendations expressed in this material are those of the authors and therefore the NRF does not accept any liability in regard thereto.

\section{References}

[1] ASM International. Eds. R. Boyer, G. Welsch and E. W. Collings, Materials Properties Handbook: Titanium Alloys. ASM International, Materials Park, Ohio, 1994.

[2] ASM International. Titanium - A Technical Guide. (2nd Ed.) ASM International, Materials Park, Ohio, 2000.

[3] ASTM International. Standard Test Method for determining Residual Stresses by the Hole-Drilling Strain-Gage Method. E837-01 ${ }^{\mathrm{e} 1}$, West Conshohocken, PA, USA, 2002. 
[4] ASTM International. Standard Practice for Conducting Force Controlled Constant Amplitude Axial Fatigue Tests of Metallic Materials. E466-96 ${ }^{\mathrm{e}}$, West Conshohocken, PA, USA, 2002.

[5] ASM International. Boyer H. E., editor. Atlas of Fatigue Curves. ASM International, Materials Park, Ohio, 1986.

[6] Radonovich, D.C., Methods of extrapolating low cycle fatigue data to high stress amplitudes. MSc thesis, University of Central Florida, Florida, USA, 2007.

[7] McGrath, P.J. and Els-Botes, A, Characterising the bending behaviour of $2 D$ laser formed Titanium (Grade 2) sheet material. Proceedings of the Light Metals Conference - Advance Metals Initiative, Johannesburg, South Africa, 2010.

[8] Churchill, S.W.and Usagi, R., A general expression for the correlation of rates of transfer and other phenomena. The American Institute of Chemical Engineers,18(6), pp. 1121-1128, 1972.

[9] Bannantine, J.A., Comer, J.J. and Handrock, J.L., Fundamentals of metal fatigue analysis. Prentice-Hall: New Jersey, 1990. 